\title{
Flexible Parylene-based Microelectrode Technology for Intraocular Retinal Prostheses
}

\author{
Damien C. Rodger ${ }^{1,2, *}$, Wen $\mathrm{Li}^{1}$, Hossein Ameri ${ }^{2}$, Aditi Ray ${ }^{2}$, James D. Weiland ${ }^{2}$, \\ Mark S. Humayun ${ }^{2}$, and Yu-Chong Tai ${ }^{1}$ \\ ${ }^{1}$ Caltech Micromachining Laboratory, California Institute of Technology, Pasadena, CA, U.S.A. \\ ${ }^{2}$ Doheny Eye Institute, Keck School of Medicine of the University of Southern California, Los Angeles, CA, U.S.A.
}

\begin{abstract}
We present the first single metal layer flexible microelectrode arrays designed for intraocular implantation that utilize parylene $C$ as their primary structural and insulating material. These electrodes are fabricated as a key component of an intraocular retinal prosthesis comprising a radio-frequency coil for power and data transfer, a packaged high lead-count telemetry-recovery and driving application-specific integrated circuit (ASIC), and a high-density epiretinal stimulating microelectrode array for the treatment of retinal degenerative blindness in humans. Electrochemical tests have demonstrated that these thin-film platinum electrodes perform as necessary for neuronal stimulation. A novel bioconformal MEMS geometry for a complete intraocular system with capsular retaining-wings that enables all the components of the system to be implanted and retained within the lens capsule and vitreous cavity of the eye is also presented. The efficacy of this geometry when compared with a previous model without capsular retaining-wings has been verified by surgical implantation in animal models.
\end{abstract}

Keywords-bioMEMS; electrode array; neural prosthesis; parylene; retinal prosthesis

\section{INTRODUCTION}

Low-resolution retinal prostheses have shown great promise in enabling subjects blind from degenerative diseases such as retinitis pigmentosa and age-related macular degeneration to perceive visual data [1]. The electrode arrays used in these recent tests consist of 16 electrodes and are placed on an approximately $5 \mathrm{~mm}$ by $5 \mathrm{~mm}$ area of the macula. Few researchers contest the hypothesis that a higher density of electrodes placed on the macula would afford the wearer a more useful visual map of the environment, likely restoring such abilities as facial recognition and coarse reading. In addition, a device that is completely implantable, with no wired connections to outside the eye, is desirable. The next-generation retinal prosthesis (Fig. 1), then, requires a high-density flexible retinal electrode array and cable, a flexible radio-frequency (RF) coil for wireless power and data recovery, and a high lead-count package for integration of these components with the driving application-specific integrated circuitry (ASIC) and discrete components, in order to establish such high-resolution macular stimulation capability. We have previously demonstrated a parylenebased drop-chip technology for scalable interconnection of ASICs and discrete components with thin-film wires [2], as

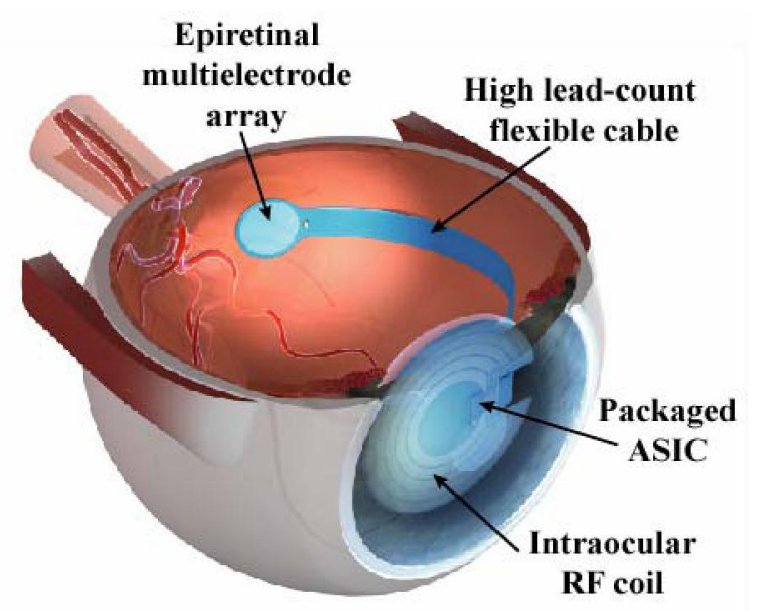

Figure 1. Next-generation retinal prosthesis comprising RF coil, packaged ASIC, flexible cable, and high-density epiretinal multielectrode array.

well as flexible parylene-based multi-metal layer RF coils for wireless telemetry [3]. Here, we discuss the fabrication and testing results for single metal layer parylene-based electrode arrays fabricated in multiple geometries. The fabrication process for the parylene electrode arrays described herein are compatible with those for our other system components. In addition, we present surgical results of an initial parylene surgical test structure that led to a novel design which improves the placement of the device within the eye. The new design has capsular retaining-wings that aid in surgical positioning.

Several research groups use parylene $\mathrm{C}$ as an outer coating for their microelectronic devices. Our goal is to use parylene as the main structural material of the retinal prosthesis in order to make full use of parylene's superior properties and minimize potential failure modes due to multiple material interfaces. The advantages of the use of parylene as the bulkmaterial for such a system, when compared with technologies based on the use of other materials such as polyimide [4] and silicon, include parylene's flexibility and mechanical strength (Young's modulus $\sim 4 \mathrm{GPa}$, in between those of silicone and polyimide), its pinhole-free conformality, its low water permeability when compared with polyimide [5], its proven intraocular [2] and United States Pharmacopeia (USP) Class 


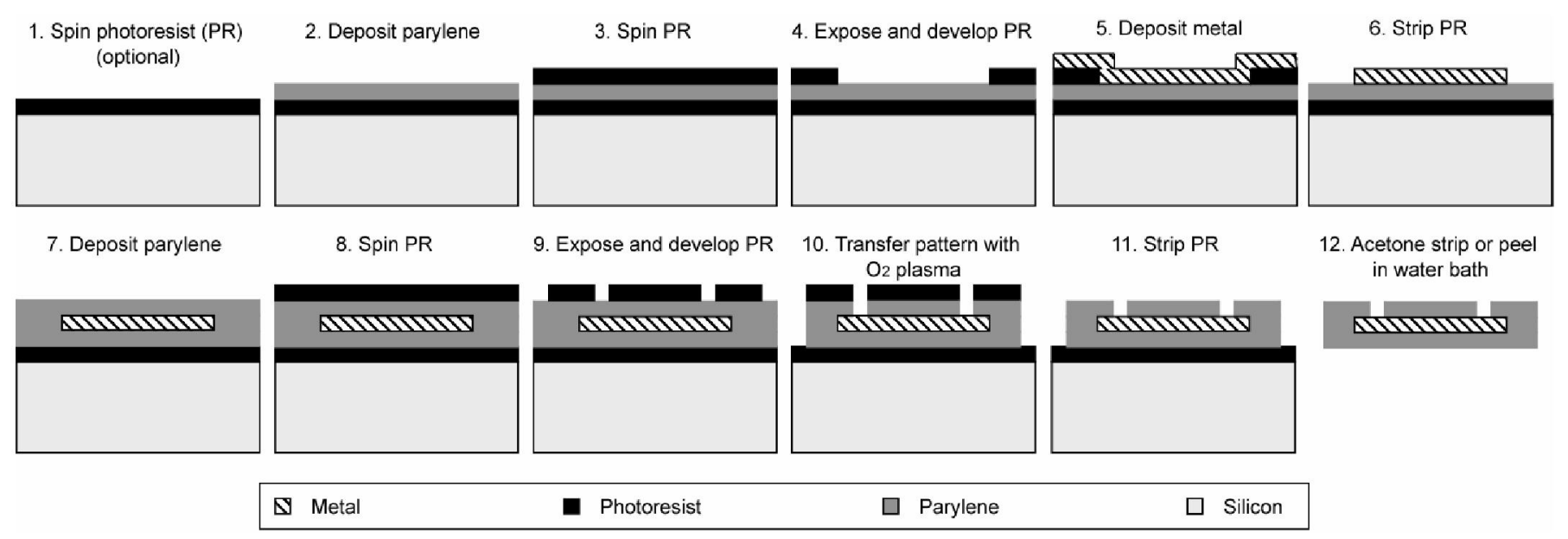

Figure 2. Fabrication process for parylene-based single-layer multielectrode arrays.

VI biocompatibility, and its transparency (which facilitates implantation as well as ongoing monitoring of the implant). In addition, because parylene is vapor-deposited in a roomtemperature chamber, parylene-based processes are post-IC compatible, facilitating high lead-count integration with ASICs. Finally, parylene can be manipulated using standard microfabrication techniques, including reactive-ion etching. These characteristics lend the material to the fabrication of high lead-count neural prosthetic devices where implementation with traditional materials would likely be problematic.

\section{FABRICATION}

Single metal layer parylene-based electrode arrays are reliably fabricated as shown in Fig. 2. A photoresist sacrificial layer is optionally spun on a standard silicon wafer. Approximately $8 \mu \mathrm{m}$ of parylene $\mathrm{C}$ is then vapor-deposited on the entire wafer. Photoresist is spun on top of the parylene, exposed in a $10 \mathrm{X}$ reduction stepper or a contact aligner (depending on the required resolution of the electrode array), and developed to achieve a liftoff pattern consisting of contacts, conductive traces, and electrodes. Approximately $200 \AA$ of titanium and $2000 \AA$ to $5000 \AA$ of metal, usually platinum, is then e-beam evaporated on the wafer. The subsequent photoresist strip generates the desired single-layer metallization pattern. An approximately $7 \mu \mathrm{m}$-thick coating of parylene $\mathrm{C}$ is then deposited, followed by a spin coating of photoresist. This photoresist etch mask is exposed over the areas of the electrodes and contact pads and to pattern the overall array geometry, and the entire wafer is then subjected to a reactive-ion etch in oxygen plasma, removing the parylene insulation over the electrodes and the parylene surrounding the array. The photoresist mask is then removed with solvent. Finally, if a sacrificial photoresist layer was used, the array is released from the substrate in an acetone bath. If no sacrificial layer was used, it is peeled from the silicon in a water bath. Fabricated electrode arrays can be roughened in oxygen plasma to increase hydrophilicity, or can be flattened or contoured through heat-treatment to ready them for implantation.

\section{RESULTS AND TESTING}

\section{A. High-density Electrode Array}

A single-layer square-grid electrode array, consisting of $256 \mathrm{Ti} / \mathrm{Pt}$ thin-film electrodes $125 \mu \mathrm{m}$ in diameter with connecting lines of $12 \mu \mathrm{m}$-pitch fabricated in the manner of Fig. 2 is shown in Fig. 3(a). A scanning electron micrograph (SEM) highlighting the typical electrode morphology in such structures is shown in Fig. 3(b). As can be seen, the parylene covering the electrode has been completely removed, whereas the incoming trace remains conformally coated with the material.

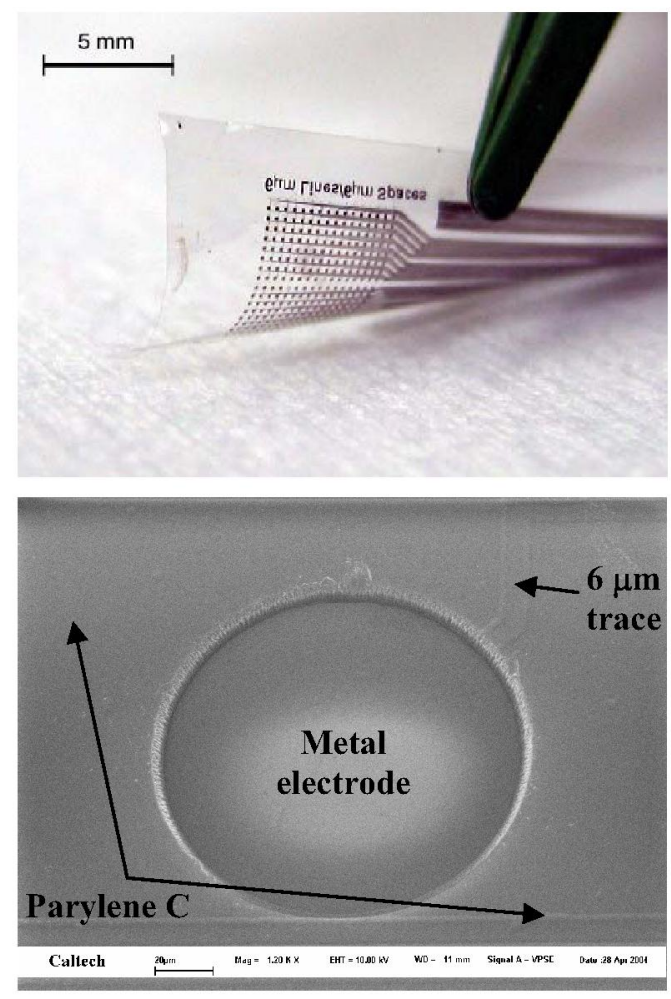

Figure 3. (a) Ti/Pt electrode array of 256 electrodes and lines of $12 \mu \mathrm{m}$-pitch; (b) typical SEM of electrode morphology. 

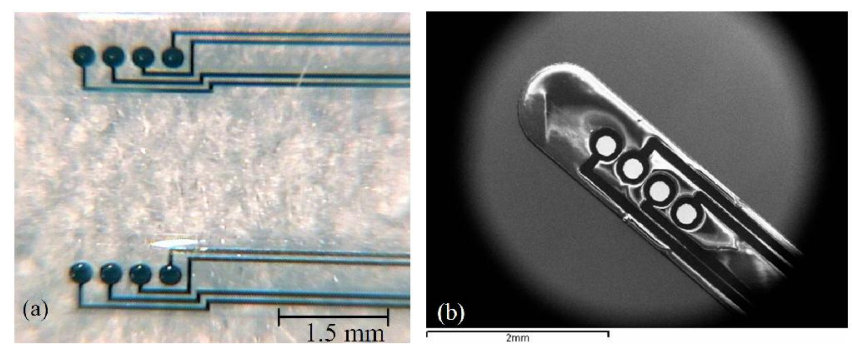

Figure 4. (a) Photograph of $50 \mu \mathrm{m}$-opening (top) and $200 \mu \mathrm{m}$-opening (bottom) test electrodes; (b) SEM of $200 \mu \mathrm{m}$ electrode array.

\section{B. Electrochemistry Test Electrodes}

Platinum test electrodes with exposed diameters of $50 \mu \mathrm{m}$ and $200 \mu \mathrm{m}$ have also been fabricated in arrays of four (photographs of both sizes seen in Fig. 4(a), scanning electron micrograph of $200 \mu \mathrm{m}$ electrode array in Fig. 4(b)) to allow for electrochemical and ongoing accelerated-lifetime saline soak testing under active pulsing. Preliminary cyclic voltammetry of a $50 \mu \mathrm{m}$-diameter electrode (Fig. 5) in $0.1 \mathrm{M}$ PBS electrolyte shows the peaks expected for a platinum stimulating electrode when compared with those found in the literature [6].

\section{Surgical Intraocular Test Devices and Implantation}

It is important to test the feasibility of surgical implantation of such parylene-based devices in animal models to determine optimal implantation technique and to establish the robustness of the devices and materials under surgical manipulation. To this end, a prototype geometry for an all-intraocular parylenebased device, simulating the overall profiles of the required components but without the fabrication complexities, was conceived (Fig. 6) and fabricated in parylene C using a photoresist mask and an oxygen reactive-ion etch. The design included an RF coil region, intended for implantation in the lens capsule, a flexible cable, to be passed through a posterior capsulotomy into the vitreous cavity, and an electrode array, designed to be attached to the retina using a retinal tack. A dummy chip was epoxied to the cable in one of the possible positions anticipated for the ASIC, partway down the parylene cable. This test structure was then implanted in several enucleated porcine eyes to evaluate surgical approach and determine the device's mechanical stability in the eye (example surgical photographs are given in Fig. 7). A significant

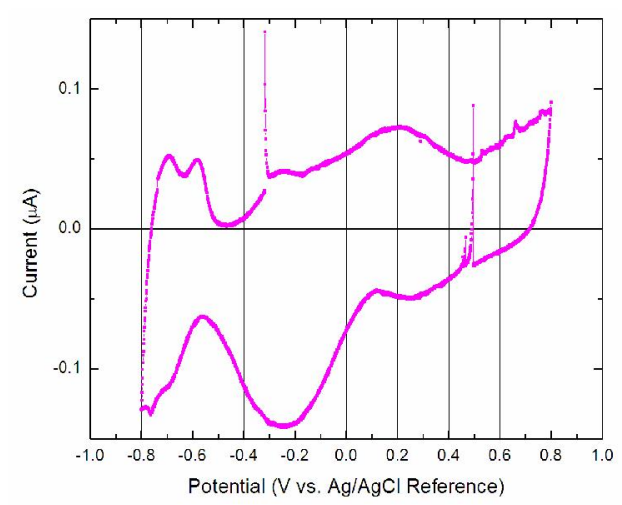

Figure 5. Cyclic voltammogram of $50 \mu \mathrm{m}$-diameter test electrode

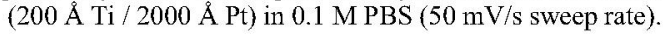

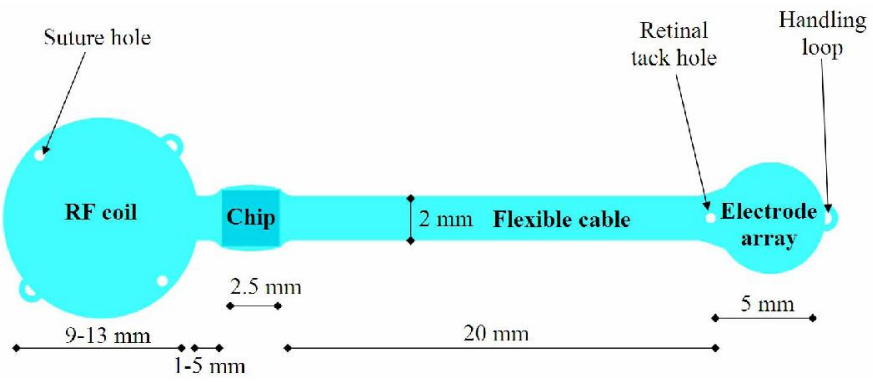

Figure 6. Prototype geometry for an all-intraocular parylene-based device.

problem with this initial design was that the flexible cable was too long, causing the cable to fold over itself and become tangled during implantation. In addition, it was discovered that the point of attachment of the flexible cable to the RF coil region caused a cabling effect, where the RF coil region tended to be drawn through the posterior capsulotomy into the vitreous cavity during surgery. To mitigate these problems, a second model has been designed (Fig. 8) that utilizes capsular retaining-wings to tether the $\mathrm{RF}$ coil region in place while the cable exits through the capsulotomy into the posterior segment. In addition, electrodes, wires, and contact pads were added to the overall structure to monitor for electrical malfunction upon implantation. To ease continuity testing, each of the four electrodes in this design was connected via two traces to large contact pads in the RF coil region. The length of the cable was also shortened to facilitate implantation in the porcine and canine animal models (it is important to note that these eyes have different average geometries than human eyes, so precise device dimensions will vary for human implantation). These
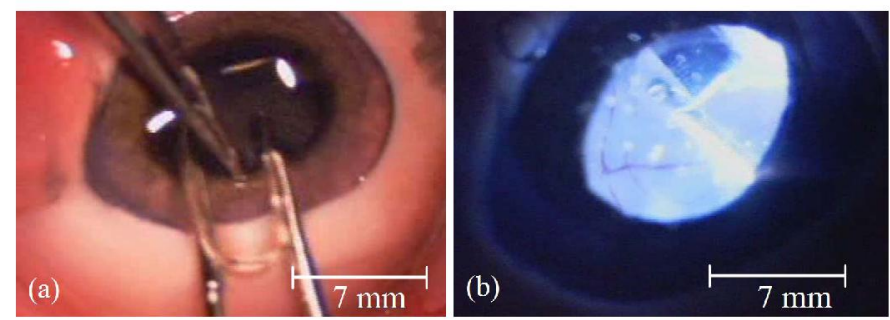

Figure 7. Example surgical photographs of prototype geometry implantation under (a) anterior and (b) intravitreal illumination.

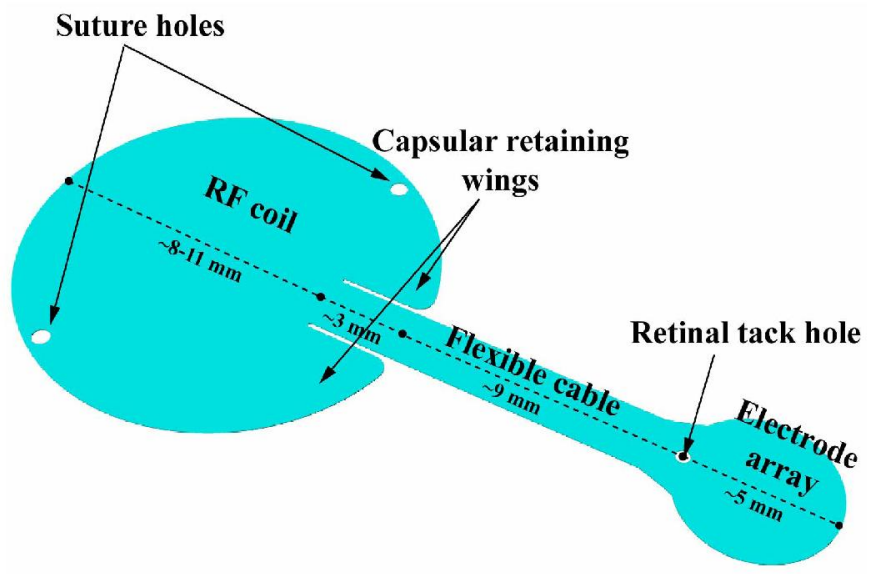

Figure 8. Bioconformal geometry for an intraocular retinal prosthesis. 


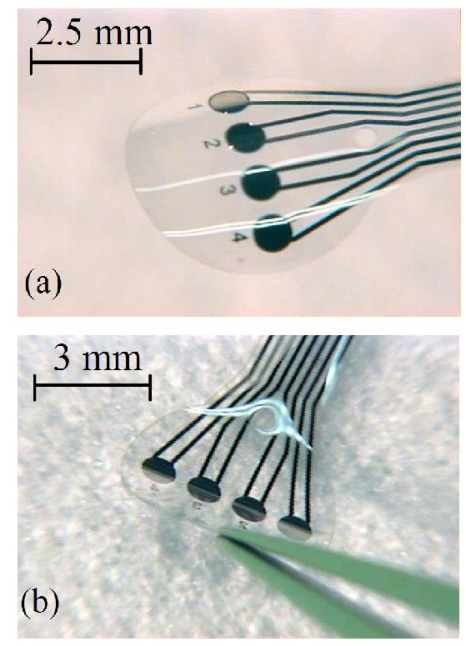

Figure 9. Fabricated implantation test structures (a) retinal aspect and (b) vitreal aspect.

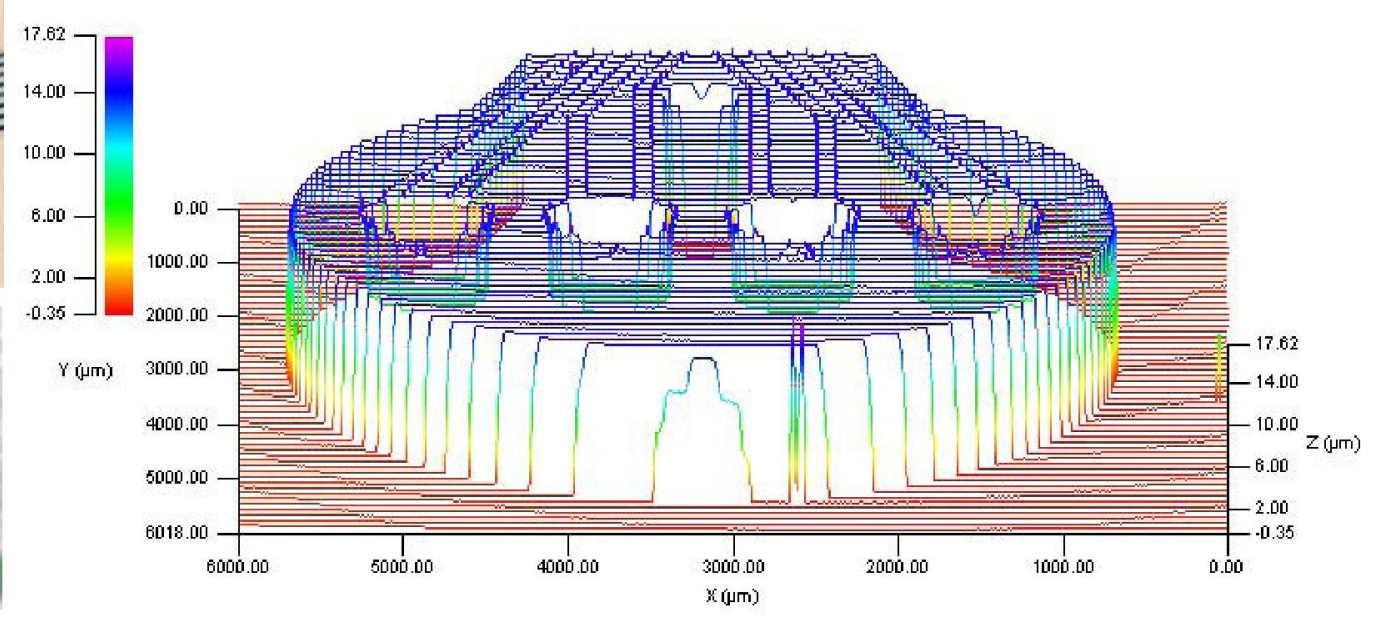

Figure 10. Surface profile of retinal aspect of electrode array region of surgical test structure. devices have been fabricated successfully (as shown in Fig. 9). The surface profile of the electrode region for this device is given in Fig. 10, showing an overall parylene thickness of approximately $14 \mu \mathrm{m}$ and electrodes recessed approximately 7 $\mu \mathrm{m}$ from the surface. Upon surgical implantation, it was noted that the design modifications resulted in a better surgical outcome and that the RF coil region shows improved anchoring in the lens capsule. Electrical tests are still underway.

\section{CONCLUSION}

Parylene-based electrode arrays with a single layer of embedded thin-film metal traces and electrodes can now be reliably fabricated and platinum test electrodes demonstrate the expected cyclic voltammetric response for platinum stimulating electrodes. In addition, prototype geometries for an allintraocular system were implanted and it has been shown that a novel geometry with capsular retaining-wings is initially successful in easing placement and improving anchoring of the device within the eye. These geometries can be implemented irrespective of the material used for the intraocular retinal prosthesis, although it is believed that parylene will ultimately prove to be the optimal material for such devices.

It is important to note that single-layer thin-film processes have an upper limit on the number of electrodes that can be reasonably accommodated within the area required for macular stimulation. This is mostly due to the need to route traces to each of the electrodes on the same plane, which often results in an electrode crowding problem if the traces are of sufficiently low impedance (i.e. not excessively narrow). Future work includes the investigation of novel fabrication methods that mitigate any such routing and electrode crowding issues.

\section{ACKNOWLEDGMENTS}

The authors especially wish to thank Mr. Trevor Roper and other members of the Caltech Micromachining Laboratory for their valuable assistance.

\section{REFERENCES}

[1] M. S. Humayun, J. D. Weiland, G. Y. Fujii, R. Greenberg, R. Williamson, J. Little, B. Mech, V. Cimmarusti, G. Van Boemel, and G. Dagnelie, "Visual perception in a blind subject with a chronic microelectronic retinal prosthesis," Vision Research, vol. 43, pp. 2573$2581,2003$.

[2] D. C. Rodger, J. D. Weiland, M. S. Humayun, and Y. C. Tai, "Scalable high lead-count parylene package for retinal prostheses," Sensors and Actuators B: Chemical, in press.

[3] W. Li, D. C. Rodger, J. Weiland, M. Humayun, and Y. C. Tai "Integrated flexible ocular coil for power and data transfer in retinal prostheses," in Proc. EMBS 2005, pp. 1028-1031.

[4] T. Stieglitz, W. Haberer, C. Lau, and M. Goertz, "Development of an inductively coupled epiretinal vision prosthesis," in Proc. EMBS 2004, pp. 4178-4181.

[5] J. J. Licari and L. A. Hughes, Handbook of Polymer Coatings for Electronics: Chemistry, Technology, and Applications, 2nd ed. Park Ridge, N.J., U.S.A.: Noyes Publications, 1990.

[6] L. S. Robblee and T. L. Rose, "The electrochemistry of electrical stimulation," in Proc. EMBS 1990, pp. 1479-1480. 